\title{
Evaluación de la co-digestión anaerobia de lodos de aguas residuales municipales con residuos de alimentos
}

\section{Anaerobic co-digestion of municipal sewage sludge with food waste}

\author{
Avaliação do co-digestão anaeróbia de lodo de \\ esgotos locais com resíduos dos alimentos
}

\author{
Ileana Consuelo Julio Guerrero*; Carlos Alberto Peláez Jaramillo²; Francisco José Molina Perez¹ \\ ${ }^{1}$ Grupo GAIA, Escuela Ambiental, Facultad de Ingeniería, Universidad de Antioquia UdeA, \\ Calle 70 No. 52-21, Medellín, Colombia. \\ ${ }^{2}$ Grupo GIEM, Instituto de Química, Facultad de Ciencias Exactas y Naturales, Universidad de Antioquia UdeA, \\ Calle 70 No. 52-21, Medellín, Colombia. \\ *ileanajulio@yahoo.es
}

Fecha Recepción: 22 de noviembre de 2015 Fecha Aceptación: 8 de enero de 2016

\begin{abstract}
Resumen
La digestión anaerobia es un proceso ampliamente utilizado para el tratamiento de los lodos producidos en las plantas de tratamiento de agua residual, debido a sus ventajas técnicas y económicas. Este artículo presenta un estudio en el que se evaluó la co-digestión de lodos de aguas residuales con residuos de alimentos (RA) como una estrategia para optimizar la digestión de los lodos. Se realizaron mono-digestiones y co-digestiones de los sustratos en condiciones mesofílicas $\left(35^{\circ} \mathrm{C}\right)$ usando reactores por lotes. Los lodos utilizados fueron: lodo primario (LP), lodo secundario espesado (LSE) y una mezcla de LP con LSE en la proporción 60:40 con base en sólidos totales (LP:LSE). Las co-digestiones se realizaron usando distintas proporciones de mezclas de los sustratos con base en sólidos volátiles totales: $L P: R A=30: 70, L P: R A=50: 50, L P: R A=70: 30$ y $(L P+L S E): R A=70: 30$. La máxima producción de metano, $0,25 \mathrm{LCH}_{4} / g S V a d i c i o n a d o$, se obtuvo para la mezcla $\mathrm{LP}: \mathrm{RA}=30: 70$, presentando una producción $32 \%$ mayor que la obtenida en la mono-digestión del lodo primario.
\end{abstract}

Palabras clave: co-digestión, producción de metano, lodo primario, lodo secundario espesado, residuos de alimentos.

\begin{abstract}
Anaerobic digestion is a process widely used for the treatment of sludge generated during wastewater treatment, due to its technical and economic advantages. This paper presents a study in which the co-digestion of sewage sludge with food waste (FW) was evaluated as a strategy for optimizing the digestion of sludge. Mono-digestions and co-digestions of the substrates were performed under mesophilic conditions $\left(35^{\circ} \mathrm{C}\right)$ using batch reactors. The sludge used were: primary sludge (PS), thickened secondary sludge (TSS), and a mixture of PS with TSS in a ratio 60:40 based on total solids (PS:TSS). The co-digestions were performed using different ratios of mixtures of the substrates based on total volatile solids: $P S: F W=30: 70, P S: F W=50: 50$, $P S: F W=70: 30$ y (PS+TSS): $F W=70: 30$. The maximum methane yield, $0,25 \mathrm{LCH}_{4} / \mathrm{gVS}$ added, was obtained for the mixture PS:FW=30:70, showing a yield $32 \%$ higher than the one obtained in the mono-digestion of PS.
\end{abstract}

Keywords: co-digestion, methane yield, primary sewage sludge, thickened secondary sludge, food waste.

Cita: Julio Guerrero IC, Peláez Jaramillo CA, Molina Perez FJ. Evaluación de la co-digestión anaerobia de lodos de aguas residuales municipales con residuos de alimentos. rev.ion. 2016;29(1):63-70. 


\section{Resumo}

A digestão anaeróbia é um processo muito utilizado para o tratamento dos lodos produzidos em estações de tratamento de esgoto, devido às suas vantagens técnicas e econômicas. Este artigo apresenta um estudo em que o co-digestão de lodo de esgotos com resíduos de alimentos (RA) foi avaliado como uma estratégia para otimizar a digestão dos lodos. Foram realizados mono-digestão e co-digestão dos substratos em condições mesofilicas $\left(35^{\circ} \mathrm{C}\right)$ utilizando reactores descontínuos. Os lodos utilizado foram: lodo primária (LP), lodos secundário espessadas (LSE) e uma mistura de LP com LSE em 60:40 à base de sólidos totais (LP:LSE). As co-digestões foram realizadas utilizando diferentes proporções de misturas de substratos à base de sólidos totais voláteis: $L P: R A=30: 70, L P: R A=50: 50, L P: R A=70: 30$ $(\mathrm{LP}+\mathrm{LSE}): \mathrm{RA}=70: 30$. A máxima produção de metano, 0,25 $\mathrm{LCH}_{4} / \mathrm{gSV}$ adicionado, foi obtido por mistura de $\mathrm{LP}: R A=30: 70$, apresentando uma produção $32 \%$ maior que a obtida no mono-digestão de lodo primário.

Palabras-chave: co-digestão, produção de metano, lodo primária, lodos secundário espessadas, resíduos de alimentos.

\section{Introducción}

El tratamiento y disposición de los lodos generados durante el tratamiento de las aguas residuales es un problema creciente, que representa hasta el $50 \%$ de los costos de operación de las plantas de tratamiento [1].

Los lodos son producto de las diferentes operaciones unitarias y de procesos realizados en las plantas de tratamiento de agua residual [2]. Los lodos denominados primarios son lodos provenientes de la sedimentación primaria utilizada para remover sólidos sedimentables que se espesan fácilmente por gravedad. Los lodos secundarios están constituidos por productos de la conversión a biomasa de los residuos solubles del efluente proveniente del tratamiento primario y partículas que escapan a este tratamiento [3]. Ambos tipos de lodos deben ser tratados antes de su disposición ya que contienen materia orgánica y patógenos [2]. Generalmente en la plantas de tratamiento de aguas residuales los lodos son tratados a través del proceso de digestión anaeróbica, ya que ésta es una tecnología económica y además permite la obtención de biogás, una fuente de energía renovable, el cual puede ser usado como combustible para compensar los requerimientos de energía de las plantas. Sin embargo, en la digestión anaeróbica de los lodos con bajo contenido de sólidos a menudo se producen bajos volúmenes de biogás [1,4]. El contenido de sólidos y el balance de nutrientes pueden ser mejorados a través de la co-digestión de los lodos con otras sustancias orgánicas tales como la fracción orgánica de residuos sólidos municipales, residuos de alimentos, residuos agrícolas y cultivos energéticos [2].
El proceso de co-digestión consiste en la digestión anaeróbica simultánea de múltiples residuos orgánicos, con características complementarias $[5,6]$. Algunos de los beneficios del proceso de co-digestión son los siguientes: aumento de la producción de biogás, dilución de compuestos potencialmente tóxicos, mejoramiento del equilibrio de nutrientes, efecto sinérgico de los microorganismos, incremento de la carga de materia orgánica biodegradable, niveles más favorables de humedad, de alcalinidad y de la relación carbono/nitrógeno en el alimento del digestor, además de compartir los costos de tratamiento de diferentes flujos de residuos en una sola instalación $[7,8,9]$.

La co-digestión anaeróbica de los lodos de aguas residuales con la fracción orgánica de residuos sólidos, puede ser una de las soluciones más viables para optimizar la eficiencia de los digestores en las plantas de tratamiento de agua residual, los cuales tienen una capacidad libre típica que puede ser aprovechada adicionando la cantidad apropiada de la fracción orgánica de residuos sólidos orgánicos [10]. Dentro de la fracción orgánica de residuos sólidos, los residuos de alimentos constituyen un sustrato atractivo para co-digerir con los lodos en los digestores, teniendo en cuenta su alto contenido de nutrientes, rápida biodegradabilidad y su disponibilidad en grandes cantidades; por otro lado, el uso de los residuos de alimentos en la co-digestión permitiría evitar su disposición en los rellenos sanitarios, impidiendo la contaminación de las aguas subterráneas, el tratamiento de lixiviados y reduciendo la emisión de gases de efecto invernadero [4]. Sin embargo, existen algunas limitaciones que deben ser controladas en la implementación de la co-digestión 
de estos residuos, entre ellas: variabilidad de los RA, debido a que los microorganismos se aclimatan a una mezcla específica, por lo tanto, cambios en dicha mezcla producirán cambios en el rendimiento del reactor; altas concentraciones de iones metálicos, que pueden causar toxicidad en la co-digestión, riesgo que es mayor con el aumento de la fracción de RA en el proceso debido a que este tipo de residuos contienen normalmente iones metálicos; rápida acidificación de los compuestos orgánicos solubles presentes en los RA, que incrementa la concentración de ácidos grasos volátiles lo que también ocasiona toxicidad en el proceso [11]. Además el digestato producido contiene altas cargas orgánicas y nutrientes (principalmente nitrógeno y fósforo), y patógenos (puesto que los patógenos pueden sobrevivir en la digestión anaerobia, especialmente en el caso de la digestión anaerobica mesofílica); por otro lado, el almacenamiento y transporte del digestato es difícil debido a su alto contenido de agua $[12,13]$. Dependiendo de las características del digestato, éste puede requerir pos-tratamiento para su uso [14]. La co-digestión anaeróbica es una tecnología bien establecida en Europa, con Alemania y Escandinavia como pioneros. El número de plantas de co-digestión está continuamente incrementándose en muchos países de Europa, nuevas plantas de lodos y residuos de alimentos se han construido recientemente en Dinamarca, Alemania y Suiza [11].

El objetivo de este estudio fue evaluar la co-digestión de lodos de aguas residuales municipales con residuos de alimentos, para lo cual se desarrollaron co-digestiones de los sustratos usando cuatro proporciones de mezclas: LP:RA=30:70, LP:RA=50:50, LP:RA=70:30 y (LP+LSE):RA=70:30, con base en sólidos volátiles totales (SV/SV). Los resultados del proceso de co-digestión fueron comparados con la mono-digestión de cada sustrato.

\section{Metodología}

\section{Sustratos}

Los lodos usados en el estudio provienen de la planta de tratamiento de aguas residuales San Fernando de la ciudad de Medellín, Colombia. La planta trata $112.000 \mathrm{~m}^{3} /$ día de aguas residuales ( $70 \%$ de agua residual doméstica y $30 \%$ de agua residual industrial). Los lodos primarios proceden de los sedimentadores primarios y los lodos secundarios espesados, provienen del proceso de espesado de lodos secundarios por centrifugación. El tratamiento secundario en la planta se realiza a través del proceso de lodos activados. El inóculo utilizado procede de los digestores anaerobios. Los procesos de lodos activados y de digestión anaerobia tienen un tiempo de retención de lodos de 2 y 20 días, respectivamente. Los residuos de alimentos empleados, fueron obtenidos en un restaurante de la Universidad de Antioquia. Tales residuos fueron triturados en una licuadora industrial para lograr homogeneidad en el sustrato.

\section{Procedimiento experimental}

Los ensayos de potencial de metano bioquímico (PMB) fueron realizados a $35^{\circ} \mathrm{C}$, en reactores por lotes, durante un periodo de digestión de 36 días, de acuerdo al protocolo sugerido por Angelidaki et al. [15]. Como reactores se utilizaron botellas de $1 \mathrm{~L}$ a las cuales se les adaptó una válvula de tres vías para monitorear la presión y cuantificar el metano producido en la digestión.

Se utilizó una relación sustrato:inóculo 0,5:1 basada en sólidos volátiles, teniendo en cuenta que grandes volúmenes de inóculo aseguran una alta actividad microbiana, bajo riesgo de sobrecarga y bajo riesgo de inhibición [10], la baja relación sustrato:inóculo trata de emular la situación real que ocurre en los digestores. El volumen ocupado por los sustratos en las botellas fue de $300 \mathrm{ml}$. Antes de iniciar los experimentos, las botellas fueron purgadas con nitrógeno gaseoso por dos minutos para asegurar condiciones anaeróbicas [16]. Durante los ensayos las botellas fueron incubadas a $35^{\circ} \mathrm{C} \pm 2^{\circ} \mathrm{C}$ y se agitaron manualmente dos veces al día. En la Figura 1 se presenta el montaje experimental empleado. Los ensayos fueron realizados por triplicado, se usaron tres blancos que contenían solo el inóculo, los cuales permitieron calcular la producción neta de metano.

Diariamente se midió la presión del biogás en el volumen libre de la botella por medio de un transductor de presión (Centrepoint Electronics, Modelo PSI-15) y el contenido de metano se determinó con un equipo caracterizador de gases (RKI Instruments, Modelo GX-2012). El volumen de metano producido se calculó utilizando la ley de gases ideales a condiciones estándar $\left(0^{\circ} \mathrm{C}\right.$ y 1atm). La producción de metano se expresó en función de los sólidos volátiles que contenían los sustratos.

Antes del proceso de digestión se analizó en las muestras: sólidos totales (ST) y sólidos volátiles 
(SV); carbono orgánico oxidable total (C), nitrógeno total Kjeldahl (NTK), pH y alcalinidad, luego de la digestión se determinaron los parámetros de ST, SV y $\mathrm{pH}$.

\section{Métodos analíticos}

Los ST, SV y $\mathrm{pH}$ se realizaron de acuerdo al Standard Methods (APHA 2012) [17]. El nitrógeno total Kjeldahl fue analizado de acuerdo a la nota de aplicación $\mathrm{N}^{\circ} \mathrm{K}-435-\mathrm{K} 370-007$, versión A, casa fabricante BUCHI y al Standard Methods (APHA 2012) [17]. El carbono orgánico oxidable total se determinó según la norma técnica colombiana NTC 5167 [18]. La alcalinidad se realizó de acuerdo a la metodología propuesta por Jenkins et al. [19] y Ripley et al. [20].

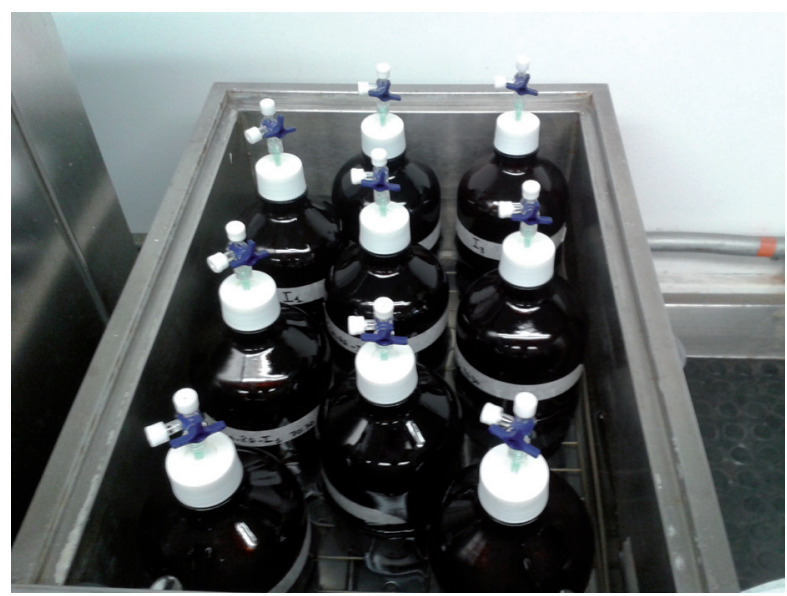

Figura 1. Montaje experimental utilizado en los ensayos de PMB

\section{Resultados y Discusión}

\section{Características de los sustratos e inóculo}

La Tabla 1 presenta los datos de la caracterización de los sustratos, del inóculo y de la mezcla LP-LSE (los valores corresponden al promedio de las diferentes caracterizaciones realizadas). El lodo primario tiene un bajo contenido de sólidos volátiles, $51,99 \%$ de los ST, y una baja relación $\mathrm{C}: \mathrm{N}, 8,86$. El lodo secundario espesado posee un contenido de materia orgánica elevado, $67,92 \%$ de los ST, debido a que este tipo de lodo está constituido principalmente por biomasa bacteriana; además el nitrógeno presente en este lodo es alto, ya que un $12 \%$ de la biomasa es nitrógeno, esto ocasiona que su relación C:N sea muy baja, 3,64. La mezcla de lodo primariolodo secundario espesado (LP:LSE=60:40 en sólidos totales), en comparación con el lodo secundario espesado presenta una mayor relación $\mathrm{C} / \mathrm{N}$, lo que es favorable para la digestión de la mezcla LP:LSE=60:40. Los residuos de alimentos tienen una mayor relación $\mathrm{C} / \mathrm{N}$ que los lodos de aguas residuales, así como un mayor contenido de materia orgánica; estas dos propiedades generan ventajas al mezclar los lodos y los residuos de alimentos.

El lodo digerido (inóculo) tiene poco carbono pues éste ha sido metanogenizado durante la digestión; también posee una elevada alcalinidad parcial, $3.563 \mathrm{mgCaCO}_{3} / \mathrm{L}$, lo que constituye una ventaja para la digestión, ya que el lodo digerido puede aportar alcalinidad a los sustratos.

Tabla 1. Características de los sustratos, del inóculo y de la mezcla LP:LSE=60:40

(valor promedio \pm desviación estándar).

\begin{tabular}{|c|c|c|c|c|c|}
\hline Parámetro & $\begin{array}{l}\text { Lodo digerido } \\
\text { (Inóculo) }\end{array}$ & $\begin{array}{c}\text { Lodo } \\
\text { primario }\end{array}$ & $\begin{array}{c}\text { Lodo secundario } \\
\text { espesado }\end{array}$ & $\begin{array}{c}\text { Residuos de } \\
\text { alimentos }\end{array}$ & LP:LSE $=60: 40$ \\
\hline Sólidos totales (\%) & $4,18 \pm 0,80$ & $4,99 \pm 0,65$ & $7,14 \pm 1,08$ & $30,63 \pm 2,27$ & $5,00 \pm 0,03$ \\
\hline Sólidos volátiles $(\%)^{\text {bs }}$ & $39,47 \pm 2,43$ & $51,99 \pm 3,13$ & $67,92 \pm 3,85$ & $95,29 \pm 0,73$ & $52,85 \pm 0,43$ \\
\hline Carbono $(\%)^{\text {bs }}$ & $14,76 \pm 1,61$ & $22,28 \pm 2,48$ & $25,04 \pm 1,96$ & $43,25 \pm 1,54$ & $24,47 \pm 0,31$ \\
\hline Nitrógeno $(\%)^{\text {bs }}$ & $4,87 \pm 0,52$ & $2,72 \pm 0,49$ & $7,02 \pm 0,66$ & $2,30 \pm 0,38$ & 3,45 \\
\hline Relación C/N & $3,15 \pm 0,32$ & $8,86 \pm 1,13$ & $3,64 \pm 0,29$ & $18,33 \pm 1,54$ & 7,10 \\
\hline Alcalinidad parcial $\left(\mathrm{mgCaCO}_{3} / \mathrm{L}\right)$ & $3.563 \pm 291$ & $195 \pm 59$ & $756 \pm 83$ & - & $611 \pm 19$ \\
\hline Alcalinidad Total $\left(\mathrm{mgCaCO}_{3} / \mathrm{L}\right)$ & $4.240 \pm 254$ & $960 \pm 151$ & $3.002 \pm 699$ & - & $1.685 \pm 0$ \\
\hline $\begin{array}{l}\text { Alcalinidad intermedia } \\
\left(\mathrm{mgCaCO}_{3} / \mathrm{L}\right)\end{array}$ & $677 \pm 173$ & $765 \pm 152$ & $2.246 \pm 616$ & - & $1.073 \pm 19$ \\
\hline $\mathrm{pH}$ & $7,60 \pm 0,14$ & $6,05 \pm 0,27$ & $6,50 \pm 0,20$ & $4,59 \pm 0,64$ & $6,40 \pm 0,07$ \\
\hline Remoción de SV (\%) & - & $20,64 \pm 0,53$ & $24,79 \pm 0,23$ & $26,62 \pm 0,99$ & $20,85 \pm 0,28$ \\
\hline $\begin{array}{l}\text { Producción de metano } \\
\left(\mathrm{LCH}_{4} / \mathrm{gSV} \text { adicionado) }\right.\end{array}$ & $0,02 \pm 0,00$ & $0,19 \pm 0,01$ & $0,19 \pm 0,01$ & $0,20 \pm 0,00$ & $0,18 \pm 0,00$ \\
\hline
\end{tabular}

bs: base seca. 
Características de las mezclas de lodos con residuos de alimentos

En las Tablas 1 y 2 se puede observar que las mezclas de lodo primario y residuos de alimentos, y la mezcla de lodo primario, lodo secundario espesado y residuos de alimentos presentan mayor cantidad de sólidos volátiles que el lodo primario, y la mezcla de lodo primario-lodo secundario espesado respectivamente, esto refleja que la adición de los residuos de alimentos a los lodos aumenta la carga orgánica disponible para la digestión.

Tabla 2. Características de las mezclas de los sustratos (valor promedio \pm desviación estándar).

\begin{tabular}{|c|c|c|c|c|}
\hline Parámetro & LP:RA $=30: 70$ & LP:RA $=50: 50$ & LP:RA $=70: 30$ & $(\text { LP+LSE) })^{*}: R A=70: 30$ \\
\hline Sólidos totales (\%) & $8,28 \pm 0,07$ & $6,55 \pm 0,06$ & $6,40 \pm 0,04$ & $5,88 \pm 0,03$ \\
\hline Sólidos volátiles (\%) & $73,39 \pm 0,25$ & $62,18 \pm 0,15$ & $60,40 \pm 0,03$ & $60,20 \pm 0,11$ \\
\hline Carbono $(\%)^{\text {bs }}$ & $31,13 \pm 1,59$ & $26,25 \pm 0,00$ & 28,88 & 28,71 \\
\hline Nitrógeno $(\%)^{\mathrm{bs}}$ & 2,79 & 2,71 & 3,09 & 3,91 \\
\hline Relación C/N & 11,14 & 9,69 & 9,35 & 7,34 \\
\hline $\begin{array}{l}\text { Alcalinidad Total } \\
\left(\mathrm{mgCaCO}_{3} / \mathrm{L}\right)\end{array}$ & $272 \pm 26$ & $797 \pm 0$ & $796 \pm 3$ & $1.250 \pm 0$ \\
\hline $\mathrm{pH}$ & $5,65 \pm 0,00$ & $5,70 \pm 0,05$ & $5,59 \pm 0,01$ & $6,16 \pm 0,03$ \\
\hline Remoción de SV (\%) & $29,04 \pm 1,12$ & $25,97 \pm 0,21$ & $22,85 \pm 0,95$ & $23,21 \pm 0,28$ \\
\hline $\begin{array}{l}\text { Producción de metano } \\
\text { ( } \mathrm{LCH}_{4} / \mathrm{gSV} \text { adicionado) }\end{array}$ & $0,25 \pm 0,01$ & $0,22 \pm 0,01$ & $0,21 \pm 0,00$ & $0,22 \pm 0,00$ \\
\hline
\end{tabular}

*La mezcla de lodo primario y lodo secundario espesado (LP:LSE) se realizó en una proporción 60:40 con base en sólidos totales. bs: base seca.

La cantidad de nitrógeno y carbono presente en los residuos es un factor crucial en la producción de biogás [21]. Una relación $\mathrm{C} / \mathrm{N}$ elevada puede generar deficiencia de nutrientes para el crecimiento y reproducción de los microorganismos; una relación $\mathrm{C} / \mathrm{N}$ muy baja puede producir inhibición de la metanogénesis debido a altas concentraciones de nitrógeno amoniacal (total o libre) [9]. El lodo primario usado en el estudio tiene una relación $\mathrm{C} / \mathrm{N}$ en el rango de 8,80 a 9,02 , al mezclarse con los residuos de alimentos, se presentó un aumento de esta relación, obteniéndose valores de 11,14, 9,69, y 9,35 para las mezclas $L P: R A=30: 70, L P: R A=50: 50$, y $L P: R A=70: 30$, respectivamente. La mezcla (LP+LSE):RA=70:30 presentó una relación $\mathrm{C} / \mathrm{N}$ de 7,34, ligeramente mayor a la relación obtenida en la mezcla de lodo primario-lodo secundario espesado. Al adicionar el inóculo a las mezclas de los sustratos, las relaciones $\mathrm{C} / \mathrm{N}$ disminuyeron debido a la alta concentración de nitrógeno del inóculo. Los valores de la relación $\mathrm{C} / \mathrm{N}$ obtenidas al adicionar los residuos de alimentos a los lodos de aguas residuales se encuentran dentro del rango que se ha reportado en la literatura, 6 a 15,4
$[14,22,23]$.

Por otro lado, a pesar de que las relaciones $\mathrm{C} / \mathrm{N}$ de las mezclas se encontraron por fuera del rango propuesto por algunos investigadores como adecuado para la digestión anaerobia (i.e., $20<\mathrm{C} / \mathrm{N}<30$ ) [24], la adición de los residuos de alimentos a los lodos de aguas residuales aumentó la producción de metano, al incrementar la relación $\mathrm{C} / \mathrm{N}$ de los lodos; la mayor producción de metano, 0,25 $\mathrm{LCH}_{4} / \mathrm{gSV}$ adicionado, se obtuvo para la mezcla $L P: R A=30: 70$, la cual presentó la mayor relación $\mathrm{C} / \mathrm{N}=11,14$.

El valor de la relación $\mathrm{C} / \mathrm{N}$ de la mezcla de lodo primario y lodo secundario tratada en los digestores anaerobios en la Planta San Fernando, está alrededor de 7. La adición de los residuos de alimentos a la mezcla LP:LSE permitiría aumentar la relación $\mathrm{C} / \mathrm{N}$ del alimento del digestor, lo que ocasionaría un incremento de la producción de metano actual de la planta; de acuerdo a los resultados obtenidos, una mezcla de $(L P+L S E): R A=70: 30$ en sólidos volátiles permite alcanzar una producción de $0,22 \mathrm{LCH}_{4} / \mathrm{gSV}$ adicionado, un valor $22 \%$ mayor que el obtenido con la mezcla de lodo primario y lodo secundario. 


\section{Producción de metano}

En la Figura 2 se observa que la producción de metano del lodo primario, lodo secundario espesado y de la mezcla lodo primario-lodo secundario espesado fue muy similar. Se obtuvo una producción de metano de $0,19 \mathrm{LCH}_{4} / \mathrm{gSV}$ adicionado para el LP y LSE, y $0,18 \mathrm{LCH}_{4} / \mathrm{gSV}$ adicionado para la mezcla LP:LSE=60:40. La producción de metano para los residuos de alimentos fue $0,20 \mathrm{LCH}_{4} / \mathrm{gSV}$ adicionado, un valor similar al obtenido para los lodos de aguas residuales; sin embargo, durante casi todo el periodo de digestión, la producción de metano de los residuos de alimentos fue menor que la de los lodos, posiblemente debido a que el inóculo usado no estaba adaptado a las características de los residuos de alimentos.

En cuanto a la producción de metano en las co-digestiones, Figura 3 , se observa que a medida que se incrementa la carga orgánica en las mezclas de lodo primario y residuos de alimentos (mayor contenido de residuos de alimentos en las mezclas) la producción de metano aumenta. La mezcla LP:RA=30:70, la cual poseía la mayor relación $\mathrm{C} / \mathrm{N}$, presentó la máxima producción de metano, 0,25 $\mathrm{LCH}_{4} / \mathrm{gSV}$ adicionado, una producción $32 \%$ mayor que la obtenida en la mono-digestión del lodo primario, además con esta mezcla se obtuvo la mejor remoción de sólidos volátiles. Siddiqui et al. [25] realizaron la digestión de residuos de alimentos con lodos de aguas residuales (mezcla de lodo primario y lodo secundario) usando diferentes mezclas de los sustratos, encontrando también que el aumento de los residuos de alimentos en las mezclas mejora el potencial de metano, al incrementar tanto la producción de biogás acumulada y la tasa de producción de metano. Por otro lado, Cabbai et al. [10] realizaron la co-digestión de lodos de aguas residuales (mezcla de lodo primario y lodo secundario) con residuos de alimentos en las proporciones 0,23gSVRA/gSVmezclalodos, y 2,09gSVRA/gSVmezclalodos (en el presente estudio se utilizaron proporciones similares, 0,43 a 2,33gSVRA/gSVmezclalodos) y obtuvieron producciones de metano de $0,29 \mathrm{LCH}_{4} / \mathrm{gSV}$ adicionado y $0,37 \mathrm{LCH}_{4} / \mathrm{gSV}$ adicionado, respectivamente. Estos valores son superiores a los obtenidos en el presente estudio, lo que posiblemente se debe a la relación inóculo:sustrato de 3 que utilizaron Cabbai et al.[10], mientras que en este estudio se utilizó una relación inóculo:sustrato de 2; la mayor relación inóculo:sustrato pudo generar una degradación más eficiente del sustrato.

0,25

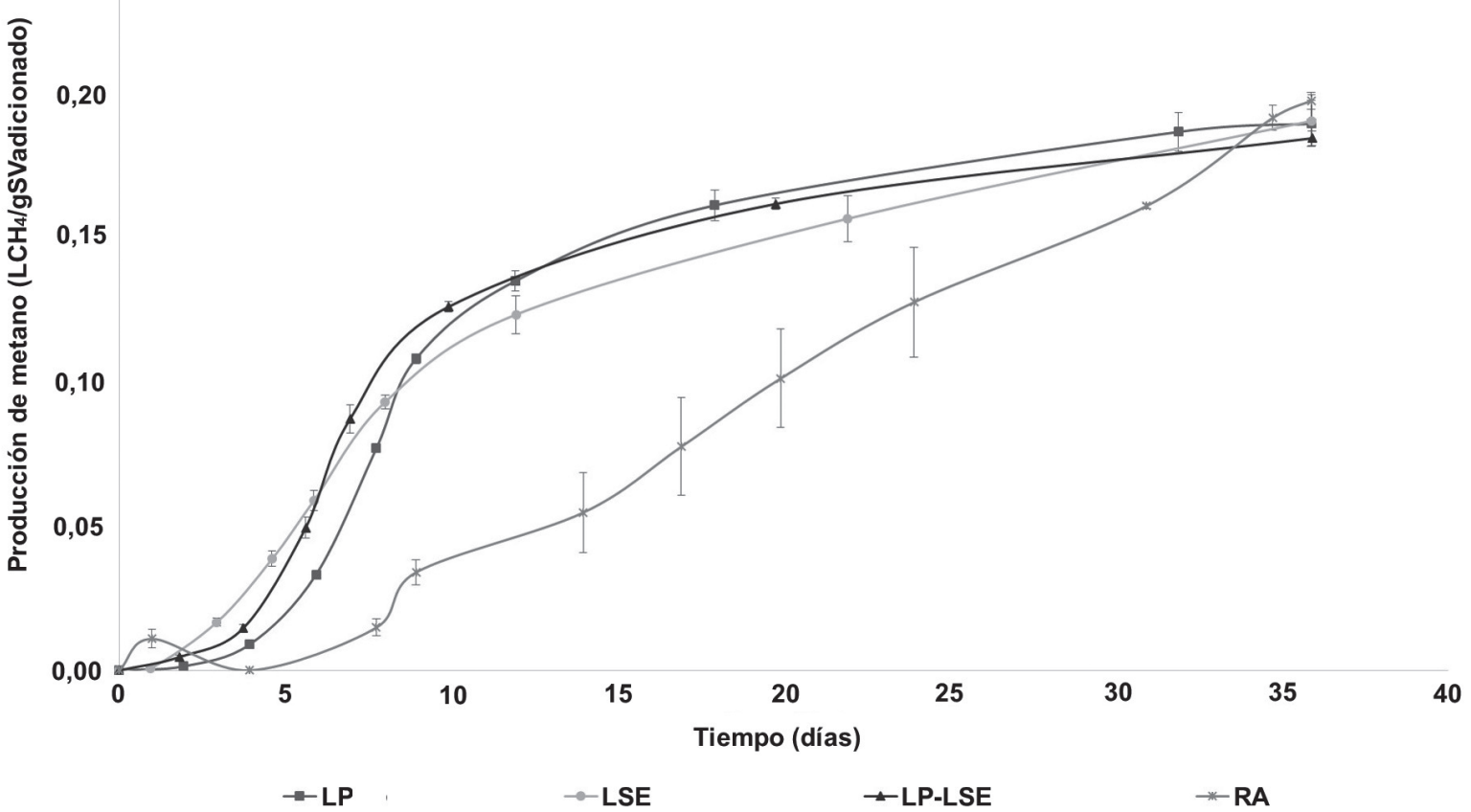

Figura 2. Producción de metano acumulada para cada sustrato y la mezcla LP-LSE. 


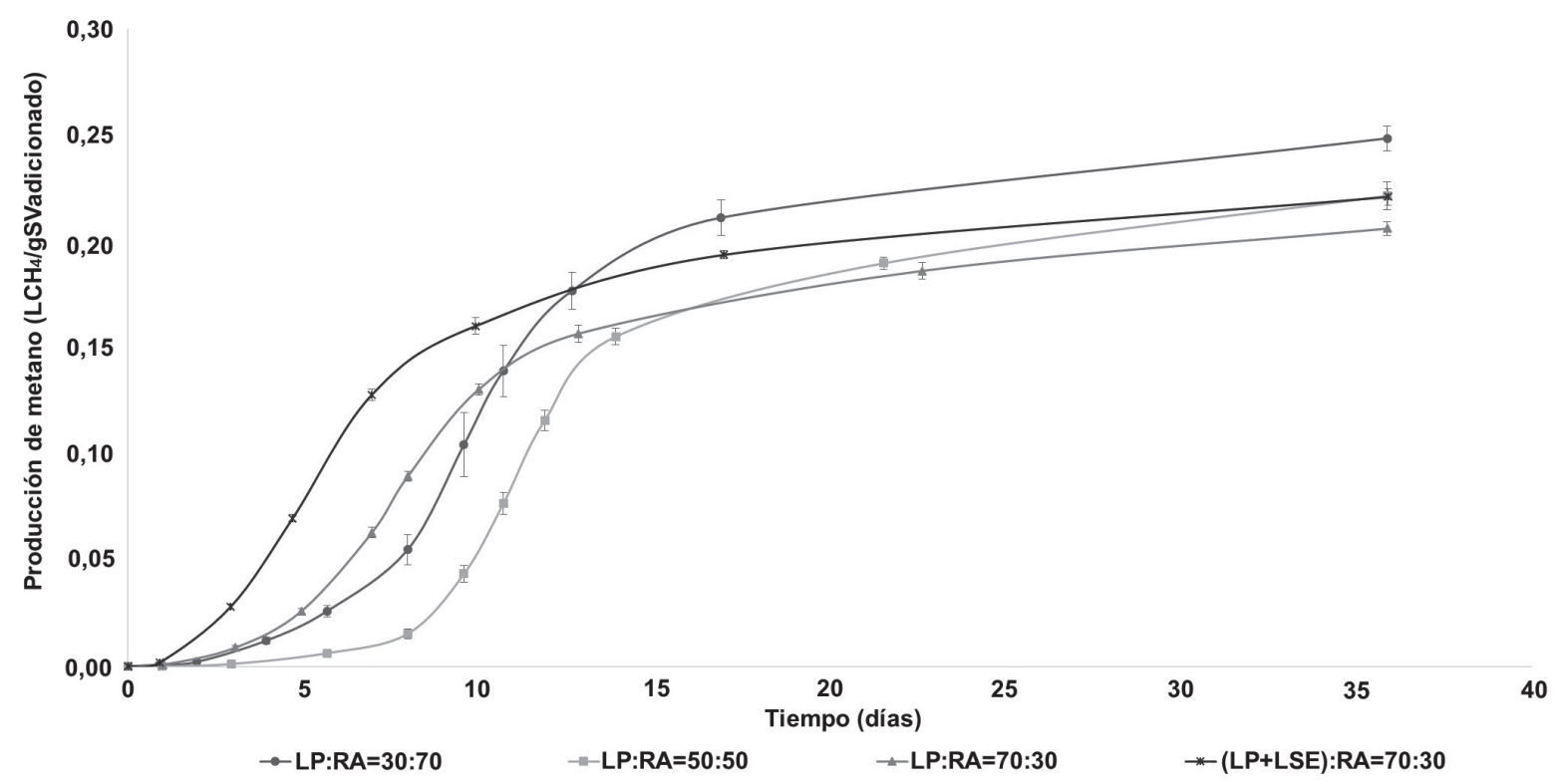

Figura 3. Producción de metano acumulada para las mezclas de lodos y residuos de alimentos.

Por otra parte, aunque la producción de metano final de las mezclas (LP+LSE):RA=70:30 y LP-RA=70:30 fue similar, durante el periodo de digestión la producción de metano para la primera mezcla fue mayor, lo que sugiere que hay un mayor efecto sinérgico al adicionar residuos de alimentos a la mezcla de lodo primario con lodo secundario espesado que al adicionar residuos de alimentos solo al lodo primario.

Durante la digestión, las mezclas $L P: R A=30: 70$ y $L P: R A=70: 30$ presentaron una fase de retardo de aproximadamente 4 días, y la mezcla LP:RA $=50: 50$ exhibió una fase de retardo de 8 días; esto se debe a que el inóculo usado (lodo digerido), no está adaptado para trabajar con la combinación de esos sustratos. En contraste, la mezcla de $(\mathrm{LP}+\mathrm{LSE}): \mathrm{RA}=70: 30$, arrancó más rápidamente la producción de metano, posiblemente debido a que ésta en su mayor proporción correspondía a una mezcla de lodo primario y lodo secundario espesado, la cual constituye el sustrato al que está adaptado el inóculo (lodo digerido) utilizado en los ensayos.

\section{Conclusiones}

La adición de los residuos de alimentos a los lodos de aguas residuales propicia el aumento de la producción de metano en la digestión, debido a que los residuos de alimentos brindan carbono orgánico a los lodos, mejorando la relación $\mathrm{C} / \mathrm{N}$ de la mezcla de sustratos. En las co-digestiones realizadas, la cantidad de metano más alta se obtuvo para la mezcla $L P: R A=30: 70$, que corresponde a la mezcla con mayor carga orgánica. Por lo tanto, la co-digestión de lodos y residuos de alimentos es una alternativa atractiva para optimizar la digestión de los lodos en las plantas de tratamiento de agua residual y aumentar la producción de metano utilizando infraestructura ya instalada.

\section{Agradecimientos}

Los autores agradecen al Área de Tratamiento de Aguas Residuales de Empresas Públicas de Medellín E.S.P. por suministrar muestras de lodo de la PTAR San Fernando e información básica para la realización de esta investigación.

\section{Referencias bibliográficas}

[1] Appels L, Lauwers J, Degrève J, Helsen L, Lievens B, Willems K, et al. Anaerobic digestion in global bio-energy production: Potential and research challenges. Renew Sustain Energy Rev. 2011;15(9):4295-301.

[2] Rao PV, Baral SS. Experimental design of mixture for the anaerobic co-digestion of sewage sludge. Chem Eng J. 2011;172(12):977-86. 
[3] Arnaiz C, Gutierrez JC, Lebrato J. Biomass stabilization in the anaerobic digestion of wastewater sludges. Bioresour. Technol. 2006;97(10):1179-84.

[4] Nathan DP, Ronald WT, Steve SH. Comparison of methane production by co-digesting fruit and vegetable waste with first stage and second stage anaerobic digester sludge from a two stage digester. Water Sci. Technol. 2012;65(7):1252-7.

[5] Kim J, Kang C-M. Increased anaerobic production of methane by co-digestion of sludge with microalgal biomass and food waste leachate. Bioresour. Technol. 2015;189:40912.

[6] Cesaro A, Naddeo V, Amodio V, Belgiorno V. Enhanced biogas production from anaerobic codigestion of solid waste by sonolysis. Ultrason Sonochem. 2012;19(3):596-600.

[7] Gómez X, Cuetos MJ, Cara J, Morán A, García Al. Anaerobic co-digestion of primary sludge and the fruit and vegetable fraction of the municipal solid wastes: Conditions for mixing and evaluation of the organic loading rate. Renew Energy. 2006;31(12):2017-24.

[8] Bond T, Brouckaert CJ, Foxon KM, Buckley CA. A critical review of experimental and predicted methane generation from anaerobic codigestion. Water Sci Technol. IWA Publishing. 2012;65(1):183-9.

[9] Lin J, Zuo J, Gan L, Li P, Liu F, Wang K, et al. Effects of mixture ratio on anaerobic codigestion with fruit and vegetable waste and food waste of China. J Environ Sci. 2011;23(8):1403-8.

[10] Cabbai V, Ballico M, Aneggi E, Goi D. BMP tests of source selected OFMSW to evaluate anaerobic codigestion with sewage sludge. Waste Manag. 2013;33(7):1626-32.

[11] lacovidou E, Ohandja D-G, Voulvoulis N. Food waste co-digestion with sewage sludge - Realising its potential in the UK. J Environ Manage. 2012;112:267-74.

[12] Huang W, Huang W, Yuan T, Zhao Z, Cai W, Zhang $Z$, et al. Volatile fatty acids (VFAs) production from swine manure through shortterm dry anaerobic digestion and its separation from nitrogen and phosphorus resources in the digestate. Water Res. 2016;90:344-53.

[13]Zeng Y, De Guardia A, Dabert P. Improving composting as a post-treatment of anaerobic digestate. Bioresour. Technol. 2016;201:293303.

[14] Zeshan, Visvanathan C. Evaluation of anaerobic digestate for greenhouse gas emissions at various stages of its management. Int Biodeterior Biodegradation. 2014;95(A):16775.

[15] Angelidaki I, Alves M, Bolzonella D, Borzacconi L, Campos JL, Guwy AJ, et al. Defining the biomethane potential (BMP) of solid organic wastes and energy crops: a proposed protocol for batch assays. Water Sci Technol. 2009;59(5):927-34.

[16] Ponsá S, Gea T, Sánchez A. Anaerobic codigestion of the organic fraction of municipal solid waste with several pure organic cosubstrates. Biosyst Eng. 2011;108(4):352-60.

[17]APHA. Standard Methods for Examination of Water and Wastewater. 22nd ed. New York: American Public Health Association; 2012.

[18] Instituto Colombiano de Normas Técnicas NTC 5167. Productos para la industria agrícola. Productos orgánicos usados como abonos o fertilizantes y enmiendas de suelo. Bogotá, Colombia; 2004.

[19] Jenkins SR, Morgan JM, Sawyer CL. Measuring anaerobic sludge digestion and growth by a simple alkalimetric titration. Water Pollut Control Fed. 1983;55(5):448-53.

[20] Ripley LE, Boyle WC, Converse JC. Improved alkalimetric monitoring for anaerobic digestion of high strength wastes. Water Pollut Control Fed. 1986;58(5):406-11.

[21] Jain S, Jain S, Wolf IT, Lee J, Tong YW. A comprehensive review on operating parameters and different pretreatment methodologies for anaerobic digestion of municipal solid waste. Renew Sustain Energy Rev. 2015;52:142-54.

[22] Sosnowski P, Wieczorek A, Ledakowicz S. Anaerobic co-digestion of sewage sludge and organic fraction of municipal solid wastes. Adv Environ Res. 2003;7(3):609-16.

[23] Koch K, Plabst M, Schmidt A, Helmreich $B$, Drewes JE. Co-digestion of food waste in a municipal wastewater treatment plant: Comparison of batch tests and full-scale experiences. Waste Manag. 2016;47(A):28-33.

[24] Marañón E, Castrillón L, Quiroga G, Fernández-Nava Y, Gómez L, García MM. Codigestion of cattle manure with food waste and sludge to increase biogas production. Waste Management.2012;32(10):1821-5.

[25] Siddiqui Z, Horan NJ, Anaman K. Optimisation of C:N Ratio for Co-Digested Processed Industrial FoodWaste and Sewage Sludge Using the BMP Test. Int $\mathrm{J}$ Chem React Eng. 2011;9(1):1-12. 